\title{
Tenascin-C Mimetic Peptide Nanofibers Direct Stem Cell Differentiation to Osteogenic Lineage
}

\author{
Melike Sever, Busra Mammadov, Mustafa O. Guler,* and Ayse B. Tekinay* \\ Institute of Materials Science and Nanotechnology, National Nanotechnology Research Center (UNAM), Bilkent University, Ankara \\ 06800, Turkey
}

\section{Supporting Information}

ABSTRACT: Extracellular matrix contains various signals for cell surface receptors that regulate cell fate through modulation of cellular activities such as proliferation and differentiation. Cues from extracellular matrix components can be used for development of new materials to control the stem cell fate. In this study, we achieved control of stem cell fate toward osteogenic commitment by using a single extracellular matrix element despite the contradictory effect of mechanical stiffness. For this purpose, we mimicked bone extracellular matrix by incorporating functional sequence of fibronectin type III domain from native tenascin-C on self-assembled peptide nanofibers. When rat mesenchymal stem cells (rMSCs) were cultured on these peptide nanofibers, alkaline phosphatase (ALP) activity and alizarin red staining indicated osteogenic differentiation even in the absence of osteogenic supplements. Moreover, expression levels of osteogenic marker genes were significantly enhanced revealed by quantitative real-time polymerase chain reaction (qRT-PCR), which showed the remarkable bioactive role of this nanofiber system on osteogenic differentiation. Overall, these results showed that tenascin-C mimetic peptides significantly enhanced the attachment, proliferation, and osteogenic differentiation of rMSCs even in the absence of any external bioactive factors and regardless of the suitable stiff mechanical properties normally required for osteogenic differentiation. Thus, these peptide nanofibers provide a promising new platform for bone regeneration.

\section{INTRODUCTION}

Recent advances in stem cell biology have shown that mesenchymal stem cells (MSCs) are pluripotent progenitor cells with the ability to differentiate into cartilage, bone, muscle, tendon, ligament, and fat cells, and they play important roles in the repair of bone defects. ${ }^{1,2}$ Differentiation of MSCs into specific and distinct phenotypes involves certain cellular transitions. ${ }^{2}$ The differentiation of MSCs in vitro largely depends on the culture conditions. The interaction of external factors, such as nutrients, structural characteristics, and bioactivity of the environment, with internal factors (genetic and epigenetic characteristics $)^{3}$ is important for the transition from one differentiation stage to another. Osteogenic differentiation of MSCs in vitro is mostly induced by the presence of osteogenic supplements including dexamethasone, ascorbic acid, and $\beta$-glycerol phosphate. ${ }^{4}$ Surface structure is also important for the behavior of the cells, ${ }^{5}$ and it directly affects the cell response ${ }^{6-8}$ and new tissue formation process. ${ }^{3,5}$ Cell fate can be manipulated through altering the rigidity of the substrate, and it was reported that osteogenic differentiation of MSCs was maximized on matrices with moduli between 10 and $40 \mathrm{kPa},{ }^{9-11}$ which emphasizes the role of physical characteristics of the matrix as regulators of stem cell behavior to guide tissue development.

The components of the extracellular matrix are important for the regulation of cell behavior during tissue development and pathology, as well as for the maintenance of normal function. ECM not only acts as a scaffold for the cells; but it also functions as a store of growth factors and cytokines. ${ }^{40}$ In bone, a large number of ECM components including collagens, proteoglycans and glycoproteins contribute to the structure of bone. $^{12,13}$ Tenascin is an extracellular matrix glycoprotein, which is also known as cytotactin or hexabrachion. The tenascin (TN) family of glycoproteins (TN-C, TN-R, TN-W, $\mathrm{TN}-\mathrm{X}$, and TN-Y) show highly dynamic patterns of expression in the embryo, especially during neural development, skeletogenesis, and vasculogenesis. They are also expressed in adults during processes including wound healing, nerve regeneration, and tissue involution, and in pathogenesis such as vascular disease, tumorigenesis, and metastasis. ${ }^{14}$ Within this family, TN-C is a hexamer of disulfide-linked subunits. Each subunit is composed of several domains of repeated structural units, which include epidermal growth factor-like repeats and fibronectin type III (FNIII) repeats. ${ }^{15} \mathrm{TN}-\mathrm{C}$ interacts with a variety of ECM molecules such as fibronectin, as well as cell surface molecules including integrins, contactin/F11, annexin II, and heparan sulfated proteoglycans, and acts as a regulator of cell behavior including adhesion, spreading and proliferation. ${ }^{16}$

Received: August 27, 2014

Revised: October 18, 2014

Published: October 24, 2014 
$\mathrm{TN}-\mathrm{C}$ is found in the condensing mesenchyme of developing bones, which is the primary center of ossification, and the periosteal and endosteal surfaces of mineralized bone. ${ }^{17,18}$ Since TN-C is found in the matrix on bone surfaces and is mostly absent in the connective tissues surrounding bone, this protein is thought to be important in the regulation of osteoblast or osteoclast function. ${ }^{19}$ It was previously shown that exogenous $\mathrm{TN}-\mathrm{C}$ is able to affect the morphology and differentiation of osteoblast-like cells and that endogenous TN-C plays an important role in the maintenance of the morphology, differentiation, and proliferation in these cells. ${ }^{19}$ In another study, a short linear amino acid sequence derived from human TN-C, VFDNFVLK, is necessary and sufficient to maintain the function of full sequence $\mathrm{TN}-\mathrm{C}$ for the formation of the neurite outgrowth. $^{20}$

The ability of self-assembled peptide amphiphile (PA) nanofibers to mimic natural ECM renders them attractive for regenerative medicine applications. PA nanofibers can be modified to meet the needs of a variety of tissues by varying the functional peptide sequences. Hydrophobic collapse of alkyl tails and $\beta$-sheet formation between PA molecules result in formation of nanofibers in aqueous solution, where short biofunctional amino acid sequences can be presented on the surface of these nanofibers. ${ }^{21}$ The supramolecular architecture of PA nanofibers enable ease of using a combination of several bioactive sequences for manipulating the cells in terms of adhesion, proliferation, and differentiation. ${ }^{22-28}$ For instance, a combination of bioactive sequences inspired by ECM proteins was used to control cell behavior for biomineralization and bone regeneration when supplemented with osteogenic culture media. $^{23}$

A tenascin-C derived peptide sequence (VFDNFVLKK) was previously shown to sufficiently maintain the function of full length TN-C protein in inducing neurite outgrowth. In the present study, we investigated whether the same sequence is also capable of inducing osteogenic differentiation based on the fact that tenascin-C is a common protein in ECM of both neural and bone tissue. Overall, we showed that tenascin- $\mathrm{C}$ mimetic self-assembled peptide nanofibers significantly enhanced the attachment, proliferation, and osteogenic differentiation of rMSCs even in the absence of any external bioactive factors and regardless of the suitable mechanical properties normally required for osteogenic differentiation. Since mechanical cues and external osteogenic supplements are known to be important requirements for osteogenic commitment of MSCs, this study presents a striking example for the ability of a single biological cue to override these requirements and makes TN-C mimetic peptide nanofibers a promising new platform for bone regeneration.

\section{EXPERIMENTAL SECTION}

2.1. Materials. All protected amino acids, lauric acid, 4- $\left(2^{\prime}, 4^{\prime}-\right.$ dimethoxyphenyl-Fmoc-aminomethyl)-phenoxyacetamido-norleucylMBHA resin (Rink amide MBHA resin), 2-(1H-benzotriazol-1-yl)1,1,3,3-tetramethyluroniumhexafluorophosphate (HBTU), and diisopropylethylamine (DIEA) were purchased from Nova-Biochem, ABCR, or Sigma-Aldrich. Calcein-AM and other cell culture materials were purchased from Invitrogen. All other chemicals and materials used in this study were purchased from Thermo Scientific or SigmaAldrich.

2.2. Synthesis of Peptide Amphiphile (PA) Molecules. Peptide amphiphile molecules were synthesized on Rink amide MBHA resin or Fmoc-Glu(OtBu)-Wang resin using the Fmoc-protected solid phase peptide synthesis method. Amino acid couplings were performed with
2 equiv of amino acids activated with 1.95 equiv of HBTU and 3 equiv of DIEA for $2 \mathrm{~h}$. Fmoc removal was performed with $20 \%$ piperidine/ dimethylformamide (DMF) solution for $20 \mathrm{~min}$. Acetic anhydride (10\%)/DMF solution was used to permanently acetylate the unreacted amine groups after each coupling step. DMF and dichloromethane (DCM) were used as washing solvents after each step. Cleavage of the PAs and protection groups from the resin was carried out with a mixture of TFA/TIS $/ \mathrm{H}_{2} \mathrm{O}$ in the ratio of $95: 2.5: 2.5$ for $3 \mathrm{~h}$. Excess TFA removal was carried out by rotary evaporation. PAs in the remaining solution were precipitated in ice-cold diethyl ether overnight. The precipitate was collected by centrifugation next day and dissolved in ultrapure water. This solution was frozen at -80 ${ }^{\circ} \mathrm{C}$ for $4 \mathrm{~h}$ and then lyophilized for 4-5 days. PAs were characterized by liquid chromatography-mass spectrometry (LC-MS) (Figure S1, Supporting Information). Mass spectrum was obtained with Agilent LC-MS equipped with Agilent 6530 Q-TOF with an ESI source and Zorbax Extend-C18 $2.1 \mathrm{~mm} \times 50 \mathrm{~mm}$ column for basic conditions and Zorbax SB-C8 $4.6 \mathrm{~mm} \times 100 \mathrm{~mm}$ column for acidic conditions. A gradient of (a) water $\left(0.1 \%\right.$ formic acid or $\left.0.1 \% \mathrm{NH}_{4} \mathrm{OH}\right)$ and $(\mathrm{b})$ acetonitrile $\left(0.1 \%\right.$ formic acid or $\left.0.1 \% \mathrm{NH}_{4} \mathrm{OH}\right)$ was used. In order to remove residual TFA, positively charged peptide amphiphiles were treated with $0.1 \mathrm{M} \mathrm{HCl}$ solution and lyophilized. To purify the peptides, an Agilent preparative reverse-phase HPLC system equipped with a Zorbax Extend-C18 $21.2 \mathrm{~mm} \times 150 \mathrm{~mm}$ column was used for basic conditions, and a Zorbax SB-C8 $21.2 \mathrm{~mm} \times 150 \mathrm{~mm}$ column was used for acidic conditions. A gradient of (a) water (0.1\% TFA or $0.1 \%$ $\left.\mathrm{NH}_{4} \mathrm{OH}\right)$ and (b) acetonitrile $\left(0.1 \%\right.$ TFA or $\left.0.1 \% \mathrm{NH}_{4} \mathrm{OH}\right)$ was used. All peptide batches were freeze-dried and reconstituted in ultrapure water at $\mathrm{pH} 7.4$ before use.

2.3. Scanning Electron Microscope (SEM) Imaging of PA Nanofiber Network. PA nanofiber networks were observed by imaging with SEM. Negatively and positively charged PA solutions (8 $\mathrm{mM}$ ) were mixed at $2: 1$ volume ratio (final volume being $50 \mu \mathrm{L}$ ) to produce gels with neutral charge. Gels were formed on silicon wafer and dehydrated by transferring to $20 \%, 40 \%, 60 \%, 80 \%$, and $100 \% \mathrm{v} / \mathrm{v}$ ethanol sequentially. They were critical point-dried by using Autosamdri 815B equipment from Tousimis. Dried PA gels were coated with $4 \mathrm{~nm} \mathrm{Au} / \mathrm{Pd}$, and SEM (FEI Quanta 200 FEG) images were taken using an Everhart-Thornley detector (ETD) at high vacuum mode at $5 \mathrm{keV}$ beam energy.

2.4. Scanning Transmission Electron Microscope (STEM) Imaging of PA Nanofiber Matrices. Samples for TEM imaging were prepared by mixing $2 \mathrm{mM}$ TC-PA or $2 \mathrm{mM}$ K-PA and $1 \mathrm{mM} \mathrm{E}$ $\mathrm{PA}$ in equal volumes and then diluting sample 10 times and placing them on a 200-mesh carbon TEM grid for 10 min followed by 2 wt \% uranyl acetate staining for 2 min and drying. STEM images at HAADF mode were acquired with FEI Tecnai G2 F30 TEM at $300 \mathrm{kV}$.

2.5. Circular Dichroism. A JASCO J815 CD spectrometer was used at room temperature. Negatively and positively charged $2.5 \times$ $10^{-4} \mathrm{M}$ PA solutions were mixed at 2:1 volume ratio (final volume being $500 \mu \mathrm{L}$ ) to produce nanofibers with net neutral charge. Measurements were carried out from 300 to $190 \mathrm{~nm}$ data interval, data pitch being $0.1 \mathrm{~nm}$, and scanning speed being $100 \mathrm{~nm} \mathrm{~min}^{-1}$, all measurements with three accumulations. Digital Integration Time (DIT) was selected as $1 \mathrm{~s}$, bandwidth as $1 \mathrm{~nm}$, and the sensitivity was standard.

2.6. Oscillatory Rheology. Oscillatory rheology measurements were performed with Anton Paar Physica RM301 rheometer operated with a $25 \mathrm{~mm}$ parallel plate configuration at $25{ }^{\circ} \mathrm{C}$. Total volume of $250 \mu \mathrm{L}$ with $8 \mathrm{mM}$ concentrations of each PA component was carefully loaded onto the center of the lower plate and incubated for $10 \mathrm{~min}$ for gelation before measurement. After equilibration, the upper plate was lowered to a gap distance of $0.5 \mathrm{~mm}$. Storage moduli $\left(G^{\prime}\right)$ and loss moduli $\left(G^{\prime \prime}\right)$ values were scanned from 100 to $0.1 \mathrm{rad} \mathrm{s}^{-1}$ of angular frequency, with a $0.5 \%$ shear strain. Three samples were measured for each PA gel.

2.7. Cell Culture and Maintenance. Rat mesenchymal stem cells (rMSCs, Invitrogen, passage number 7) were used in all cell culture experiments including viability, proliferation, gene expression analysis, ALP activity, and calcium deposition. Cells were cultured in $75 \mathrm{~cm}^{2}$ 


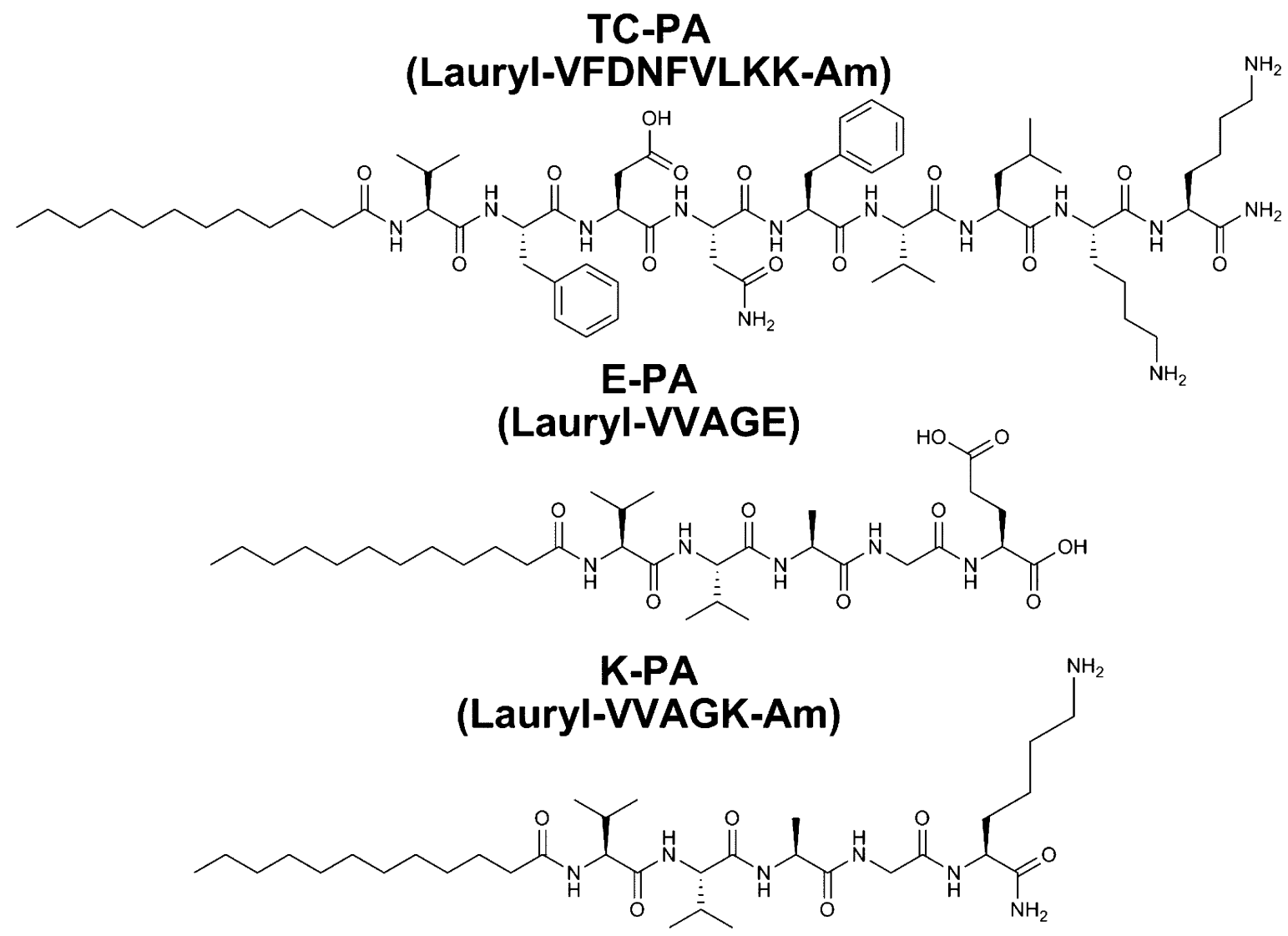

Figure 1. Self-assembled peptide amphiphile nanofibers. Chemical structures of TC-PA, E-PA, and K-PA are given. All PA molecules had a hydrophobic alkyl tail consisting of lauric acid. While TC-PA had bioactive sequence, VFDNFVLK, epitope free PA molecules carried a $\beta$-sheet forming peptide sequence, VVAG. This sequence consists of four nonpolar amino acid residues, among which valine has a very high $\beta$ sheet-forming propensity provided by its hydrophobic side chain, while glycine is used as a spacer between the hydrophobic part of the sequence and hydrophilic epitope region. ${ }^{38,39}$

flasks at a density of $2 \times 10^{3}$ cells $/ \mathrm{cm}^{2}$ at $37{ }^{\circ} \mathrm{C}$ in a humidified incubator and supplied with $5 \% \mathrm{CO}_{2}$. Cells were maintained in Dulbecco's modified Eagle's medium (DMEM) supplemented with $10 \%$ fetal bovine serum (FBS), $1 \%$ penicillin/streptomycin, and $2 \mathrm{mM}$ L-glutamine. All cell culture experiments were carried out after reaching $90 \%$ confluency. The culture medium was changed every $3-4$ days. Cells were seeded with the same medium content. The day after seeding cells, cell medium was replaced with minimum essential medium (MEM) with $3 \% \mathrm{FBS}, 1 \%$ penicillin/streptomycin, and $2 \mathrm{mM}$ L-glutamine. For alizarin red staining, this medium was also supplemented with $10 \mathrm{mM} \beta$-glycerophosphate, differently from the other experiments. Medium was changed every 3 days.

2.8. Viability Assay. To form TC-PA/E-PA or K-PA/E-PA gel, equal volumes of $3 \mathrm{mM}$ TC-PA or K-PA and $1.5 \mathrm{mM}$ E-PA were used in order to neutralize the charges. PA-gel coated plates were incubated at $37{ }^{\circ} \mathrm{C}$ for $30 \mathrm{~min}$, prior to overnight incubation in a laminar flow hood at room temperature for solvent evaporation. The next day, PA matrix formed on the plates was UV sterilized. By addition of cells in culture media to PA-coated surfaces, rehydration of the PA matrix formed a thin-gel on the surface. The viability tests of mesenchymal stem cells were performed at predetermined time intervals $(24,48,72$ h) by calcein-AM and ethidium homodimer 1 (EthD-1) staining and Alamar blue assay. Cells were incubated on PA-coated and uncoated tissue culture plates at a density of $2 \times 10^{3}$ cells $/ \mathrm{cm}^{2}$. After 24,48 , and $72 \mathrm{~h}$ of incubation, cell medium was discarded; cells were washed with PBS and then incubated with $2 \mu \mathrm{M}$ calcein-AM and $2 \mu \mathrm{M}$ EthD- 1 in PBS for $30 \mathrm{~min}$ at room temperature. Finally, five random images were taken at $10 \times$ magnification from each well for both qualitative and quantitative analysis by using a fluorescence microscope. Cells were counted with NIH Image software for proliferation. For Alamar blue assay, medium was discarded after 24,48 , and $72 \mathrm{~h}$ of incubation and replaced with medium containing 10\% Alamar blue. Blank group contained only Alamar blue medium without cells. After $4 \mathrm{~h}$ incubation at $37^{\circ} \mathrm{C}$, absorbance measurement was performed by Spectramax M5 microplate reader at 570 and $600 \mathrm{~nm}$ as reference.

2.9. SEM Imaging of Mesenchymal Stem Cells on PA Nanofiber-Coated Surfaces. The morphology and spreading of mesenchymal stem cells were examined by SEM imaging by using an ETD detector at high vacuum mode at $5 \mathrm{keV}$ beam energy. For this purpose, glass coverslips were coated with PAs, and cells were seeded on top of the coated and uncoated surfaces at a density of $2 \times 10^{3}$ cells $/ \mathrm{cm}^{2}$. Seven and twelve days after incubation, cells were rinsed with PBS and fixed with $2 \%$ gluteraldehyde/PBS and 1 wt $\% \mathrm{OsO}_{4}$ for $1 \mathrm{~h}$ each, respectively. Fixed cells were washed with water and then dehydrated sequentially in $20 \%, 40 \%, 60 \%, 80 \%$, and $100 \%$ ethanol. Samples were critical point dried with Autosamdri-815B Tousimis and coated with $6 \mathrm{~nm} \mathrm{Au}-\mathrm{Pd}$ before imaging.

2.10. Gene Expression Analysis. Gene expression profiles for differentiation were assessed by quantitative RT-PCR analysis. rMSCs were seeded at a density of $2 \times 10^{3}$ cells $/ \mathrm{cm}^{2}$ on peptide nanofiber coated surfaces and bare surface, and after 12 days of incubation of the cells on PA-coated surfaces and TCP, RNA was isolated using TRIzol (Invitrogen) according to manufacturer's instructions. Yield and purity of extracted RNA were assessed by Nanodrop 2000 (Thermo Scientific). Primer sequences were designed using NCBI database (Table S1, Supporting Information). cDNA synthesis from RNA and qRT-PCR were performed using SuperScript III Platinum SYBR Green one-step qRT-PCR kit according to manufacturer's instructions. Reaction conditions were briefly as follows: $55^{\circ} \mathrm{C}$ for $5 \mathrm{~min}, 95^{\circ} \mathrm{C}$ for 5 min, 40 cycles of $95^{\circ} \mathrm{C}$ for $15 \mathrm{~s}, X{ }^{\circ} \mathrm{C}$ for $30 \mathrm{~s}$ (varies according to primer sets), and $40^{\circ} \mathrm{C}$ for $1 \mathrm{~min}$, followed by a melting curve analysis to confirm product specificity. The reaction efficiencies for each primer 


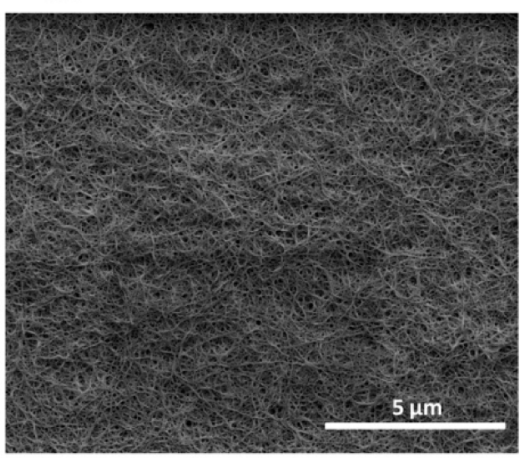

(c)

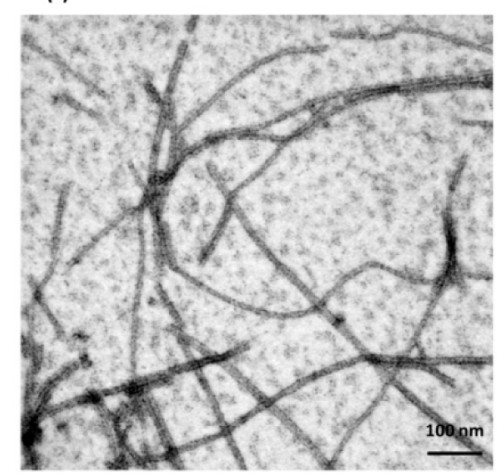

(f)

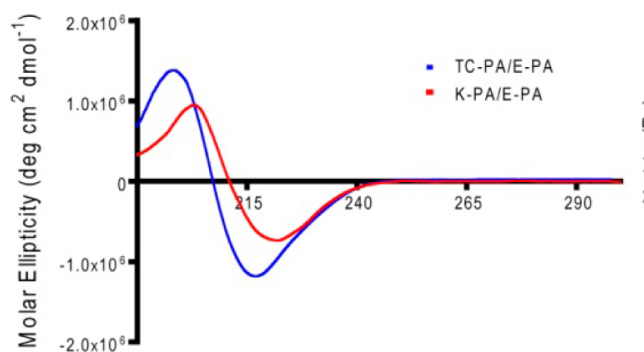

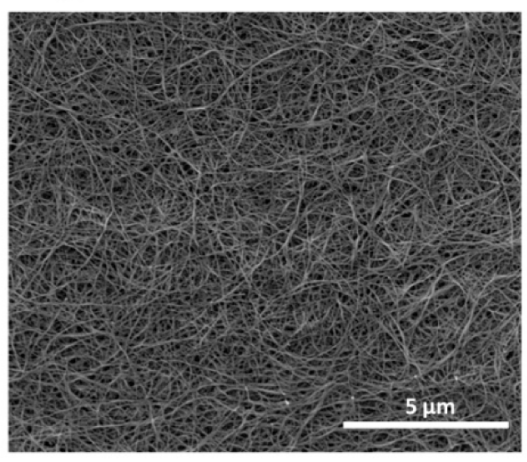

(d)

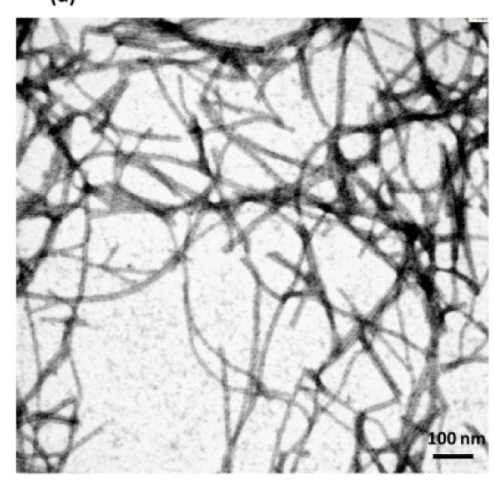

(g)

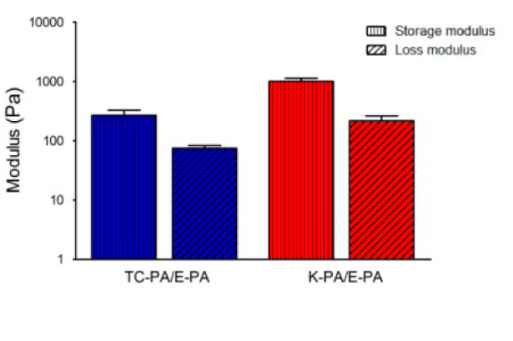

Figure 2. SEM images of TC-PA/E-PA (a) and K-PA/E-PA (b) gels reveal the ECM-like morphology of PA scaffolds. Scale bars are $5 \mu \mathrm{m}$ in length. STEM images of the TC-PA/E-PA and K-PA/E-PA nanofibers formed at $\mathrm{pH} 7.4$ are given in panels $\mathrm{c}$ and d, respectively. Scale bars are $100 \mathrm{~nm}$ in length. Characterization of secondary structure of peptide nanostructures by circular dichroism is given in panel f. Both PA combinations were found to have $\beta$-sheet secondary structure by circular dichroism analysis. Mechanical properties of PA gels are given in panel g. Rheology results showed gelation as a result of nanofibrous network formation by TC-PA/E-PA and K-PA/E-PA at $\mathrm{pH} 7.4$.

set were evaluated by plotting a standard curve using 5-fold serial dilutions of total RNA. For analysis of the expression data, primary gene expression data was normalized by the expression level of GAPDH. A comparative Ct method (Pfaffl method) was used to analyze results.

2.11. Alkaline Phosphatase (ALP) Activity Assay. ALP activity of rMSCs was analyzed by measuring the colorimetric product of $p$ nitrophenol from endogenous ALP reaction after 3 and 7 days of culture in differentiation medium. Cells $\left(3 \times 10^{3} \mathrm{~cm}^{-2}\right)$ were seeded on PA-coated and uncoated TCP surfaces. Medium change was done every 3 days. At predetermined time points, cells were rinsed with PBS, and protein extraction was performed by using M-PER protein extraction kit (Thermo) $/ 5 \%$ protease inhibitor solution as $150 \mu \mathrm{L} /$ well for $30 \mathrm{~min}$ on shaker. After centrifugation of samples at $14000 \mathrm{~g}$ for $10 \mathrm{~min}$ at $4{ }^{\circ} \mathrm{C}$, supernatants that contain proteins were taken, and BCA protein assay was performed to quantify protein amount as described in manufacturer's protocol. Absorbance was determined at $562 \mathrm{~nm}$ by using a microplate reader. For ALP activity, $50 \mu \mathrm{L}$ of protein sample was incubated with $150 \mu \mathrm{L}$ of $p$-nitrophenol phosphate substrate in 96-well plates for $30 \mathrm{~min}$ on shaker. Serial dilutions of $p$ nitrophenol in $0.25 \mathrm{M} \mathrm{NaOH}$ were used as standards. Finally, optical density was determined at $405 \mathrm{~nm}$ by using Spectramax M5 microplate reader. ALP results were normalized to total protein amount.

2.12. Imaging Mineral Deposition by Alizarin Red Staining. The ability of mineralized nodule formation and calcium deposition of MSCs on PA nanofiber-coated and uncoated tissue culture plates were assessed by using alizarin red-S staining as described previously. ${ }^{29}$ In brief, cells were incubated for 7 and 12 days at the densities of $4 \times 10^{3}$ cells $/ \mathrm{cm}^{2}$. Cells were washed with PBS and fixed with ice-cold ethanol for $1 \mathrm{~h}$ at room temperature. Then, fixed cells were washed with distilled water and stained with $40 \mathrm{mM}$ alizarin red-S solution $(\mathrm{pH}$ 4.2) for $30 \mathrm{~min}$ at room temperature on shaker. After washing 4-5 times with distilled water to get rid of nonspecific binding, PBS was added and the calcium nodules were imaged under a microscope. For quantification of calcium amount, PBS was discarded and 10\% cetylpyridinium chloride was added and incubated for $30 \mathrm{~min}$ at room temperature. At the end of incubation, solution was transferred to $96-$ well plate and absorbance measurement was done at $562 \mathrm{~nm}$. Cetylpyridinium chloride was used to release the remaining calciumbound alizarin red $S$.

2.13. Statistical Analysis. All quantitative values are presented as mean \pm SEM (standard error of means), and all the groups in 
experiments were performed with at least three replicates. Two-way analysis of variance (ANOVA) was used for statistical analysis, and $p$ value of less than 0.05 was considered statistically significant.

\section{RESULTS AND DISCUSSION}

3.1. Characterization of Peptide Amphiphile Nanofibers. In this study, osteogenic scaffolds were generated through self-assembly of peptide amphiphile molecules that carry natural extracellular matrix protein tenascin-C (TN-C) derived epitope (lauryl-VFDNFVLKK-Am (TC-PA)). ${ }^{20}$ The control nanofibers did not display this epitope ((laurylVVAGE-OH (E-PA) and lauryl-VVAGK-Am (K-PA)) (Figure 1a-c). TC-PA molecules formed nanofibers through selfassembly when mixed with oppositely charged E-PA due to electrostatic interactions, hydrophobic collapse, and $\beta$-sheet driving units at physiological $\mathrm{pH}^{30}$ Positively charged K-PA and negatively charged E-PA molecules were mixed to induce formation of nanofibers as an epitope-free control. STEM imaging showed that both TC-PA/E-PA and K-PA/E-PA nanofibers were uniform in diameter $(10-20 \mathrm{~nm})$ and several micrometers in length (Figure $2 \mathrm{c}, \mathrm{d}$ ). These nanofibers formed bundles that formed nanofiber networks that can encapsulate water to form hydrogels. These nanofiber networks resembled the nanofibrous structure of natural ECM at physiological $\mathrm{pH}$ as evidenced by SEM imaging (Figure 2a,b). The secondary structures of the tenascin- $\mathrm{C}$ mimetic and control nanofibers were characterized using circular dichroism (CD) spectroscopy. Both nanofiber types demonstrated predominantly $\beta$-sheet structure with a chiral absorbance maximum at around $200 \mathrm{~nm}$ and minimum at around $220 \mathrm{~nm}$ (Figure 2f).

Physical characteristics of the matrix are known to be a potent cue for stem cell differentiation. Mechanical properties of the gels were compared by using frequency sweep rheology measurements at constant strain. Each gel (TC-PA/E-PA gel and K-PA/E-PA gel) had higher storage modulus $\left(G^{\prime}\right)$ than loss modulus $\left(G^{\prime \prime}\right)$, indicating gel formation at physiological $\mathrm{pH}$ (Figure $2 \mathrm{~g}$ ). The elastic modulus of TN-C mimetic PA scaffold is around $270 \mathrm{~Pa}$, which is lower than the elasticity of hard matrices used to induce osteogenic differentiation in vitro ${ }^{9-11}$ and relatively lower than the elasticity modulus of epitope-free control scaffold, which was around $1000 \mathrm{~Pa}$.

3.2. PA Nanofibers Promote Cell Adhesion and Spreading. Cellular viability of rMSCs seeded on tenascin-C mimetic peptide nanofibers was assessed by calcein AM staining, by comparison to cells that were cultured on a bare glass surface and epitope-free peptide nanofibers at varying time points $(24,48$, and $72 \mathrm{~h})$. Although a lower viability was observed on TN-C mimetic PA scaffold at early time points, cells adapted to the surface after $48 \mathrm{~h}$. MSCs are multipotent cells that are known to present a nonhomogeneous cell population. Although the cells that we used were previously selected to express certain MSC markers (Invitrogen), cultured MSCs display variable phenotypes like proliferation capacity and viability as well as giving different response to differentiation stimuli. The plastic properties of MSCs can also contribute to heterogeneity of MSC cultures. ${ }^{31}$ Cellular heterogeneity within the population can be caused by persistent cell individuality coming from fluctuations of protein levels, which induce nongenetic cell individuality. ${ }^{32}$ Heterogeneity of MSC cultures may be the reason for differences in viability of our cells on tenascin-C mimetic scaffold at different time points. Although a group of the cells died up to $48 \mathrm{~h}$, viability of the cells on all surfaces was comparable at the end of $72 \mathrm{~h}$ with no significant differences between groups (Figure 3 ). This indicates that this bioactive material is causing differential

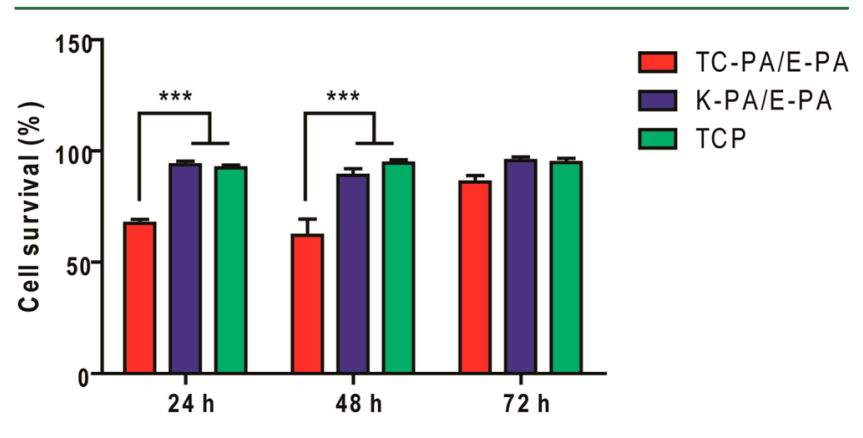

Figure 3. Viability of rMSCs when cultured on peptide nanofibers and TCP analyzed by calcein ethidium homodimer live-dead assay. This assay showed that rMSCs adapted to PA scaffolds and have comparable viability with bare surface at the end of $72 \mathrm{~h}$.

effects on different cell groups. SEM images of cells seeded on the scaffold revealed that rMSCs spread and attained their different morphologies on all of the surfaces on day 7 and 12 (Figure 4). These results indicated that tenascin-C mimetic peptide nanofibers provide attachment and spreading of rMSCs. Also, SEM images indicated that rMSCs on tenascinC mimetic scaffold display a characteristic morphology that is different from that of the cells seeded on control peptide nanofibers and bare surfaces, possibly due to the effect of $2 \mathrm{D}$ microenvironment on differentiation process.

3.3. Peptide Nanofibers Enhance Gene Expressions of Osteogenic Markers. To understand whether the morphological change of rMSCs on tenascin-C mimetic scaffold is caused by osteogenic differentiation, gene expression profiles of cells cultured on peptide network coated and bare surfaces were analyzed. First, expression of MSC markers including CD44 and CD90 were studied to verify the differentiation of MSCs. Although decrease in CD44 expression is not significant, decrease in CD90 was found to be statistically significant, confirming the loss of MSC characteristics of the cells (Figure 5a). In order to check the effect of biologically active peptide nanofiber systems on the progression of osteogenic differentiation of rMSCs at mRNA level, expression of osteogenic markers including Runx2, osteopontin, and collagen I was examined with Sox9 on day 12 (Figure 5b). Although Sox9 is the best-known master regulator for chondrocyte differentiation and cartilage formation, ${ }^{33}$ it is specifically expressed in the osteogenic cell compartments together with osteogenic markers such as Runx2, collagen I, and osteopontin. ${ }^{34}$ Expressions of Sox 9 and Runx 2 genes, early markers of ostegenic differentiation, were upregulated by $\sim 2.0$ - and 1.6-fold, respectively, when rMSCs were cultured on TC-PA/E-PA compared with cells on K-PA/E-PA and TCP surfaces. Osteopontin is a secreted adhesive glycoprotein detected within bone extracellular matrix. Osteopontin expression of the cells on TN-C mimetic scaffold was also increased, by about 3-fold. Collagen I is an important component of bone extracellular matrix, which interacts with cell surface integrins and other ECM proteins. This protein plays an important role in cell adhesion, proliferation, and differentiation of the osteoblast phenotype. In parallel to osteopontin expression, collagen I expression was also significantly upregulated in cells cultured on TC-PA/E-PA scaffold (4.5- and 1.9-fold increase when compared with K-PA/ E-PA and TCP, respectively). These significant differences in 

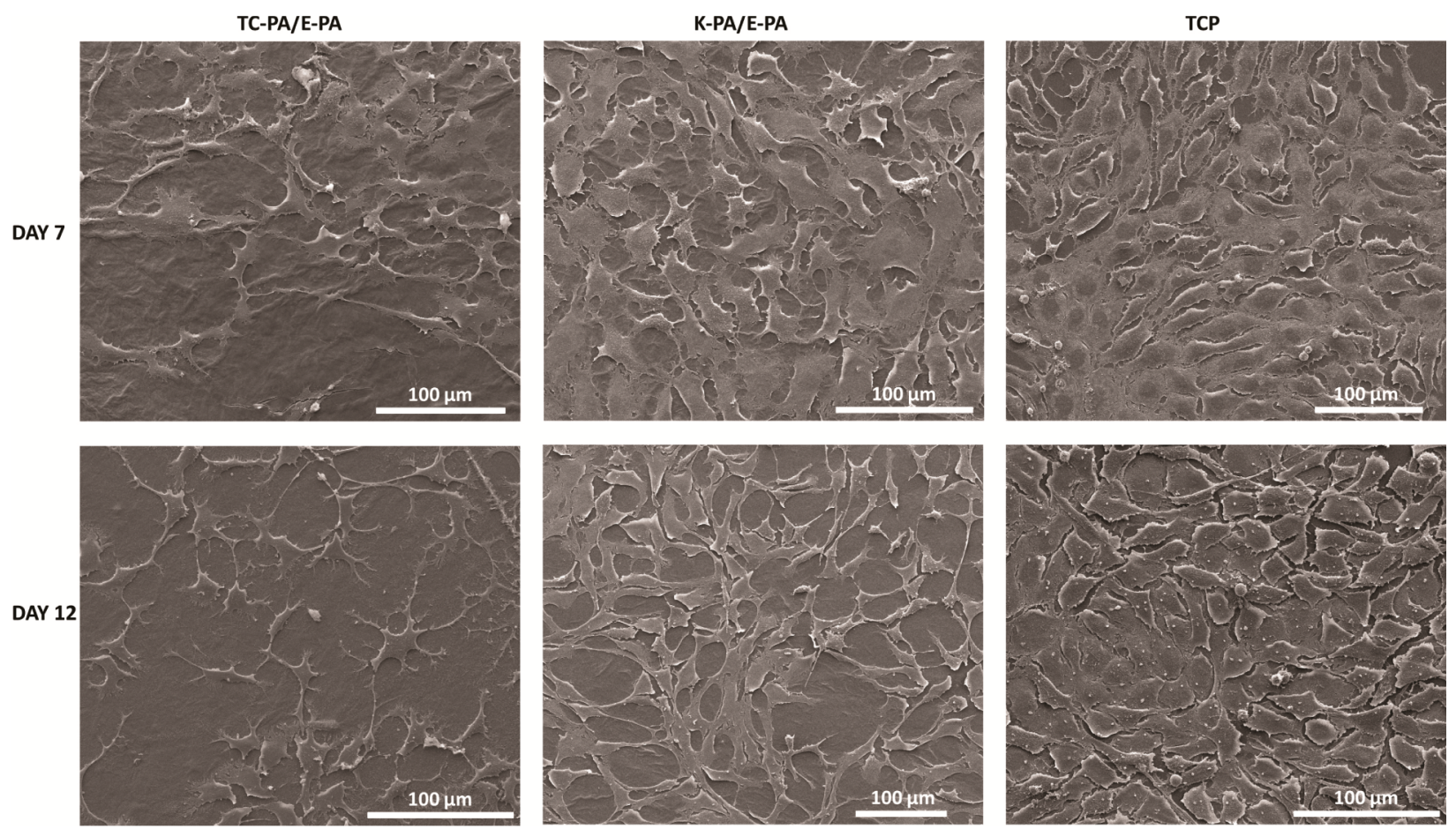

Figure 4. PA substrates are biocompatible and support adhesion of rMSCs. SEM images of rMSCs cultured on TC-PA/E-PA, E-PA/K-PA gels, and TCP at 7 days and 12 days after cell seeding. Scale bars are $100 \mu \mathrm{m}$.
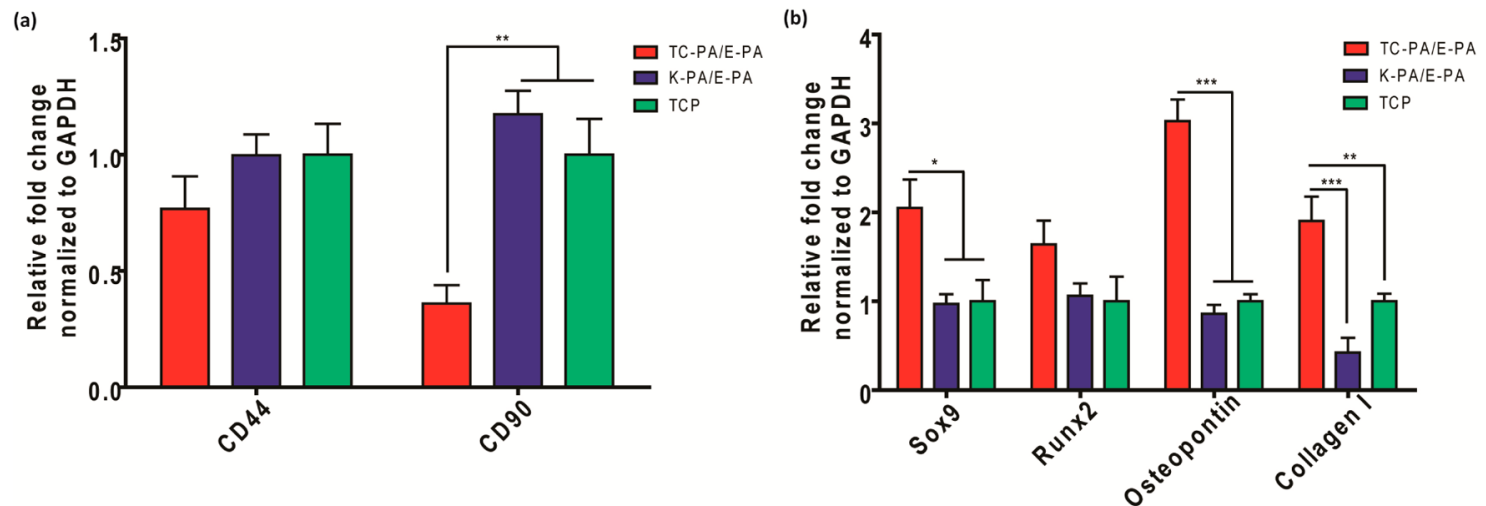

Figure 5. Gene expression analysis of (a) MSC markers and (b) osteogenic markers. The expression level of each gene was normalized against TCP and GAPDH was used as the internal control. Values represent mean \pm SEM $\left(* * * p<0.001, * * p<0.01, *_{p}<0.05\right)$.

the mRNA expression of ostegenic markers between the cells seeded on the tenascin- $\mathrm{C}$ mimetic peptide nanofiber scaffold and epitope free scaffold, as well as bare surface, were considered as evidence of osteogenic differentiation. It is widely known that MSCs have capacity to differentiate into a variety of cell types. Therefore, to be sure that the differentiated cells we observed belong to osteogenic lineage and not other cell types, we analyzed expression of specific markers of other common cell types that MSCs can differentiate. Collagen II expression was analyzed for chondrogenic differentiation along with Pax7 for myogenic differentiation, FSP for fibroblast differentiation, $\beta$ III-tubulin, NFL, and SYN1 for neural differentiation, and MBP, BLBP, and Pax6 for astroglial differentiation (Figure S5, Supporting Information). There was no detectable up-regulation in these markers indicating that rMSCs underwent osteogenic lineage commitment.

3.4. Peptide Nanofibers Stimulate Alkaline Phosphatase Activity and Mineralization. The osteoblasts are the differentiated bone-forming cells responsible for secreting collagen-proteoglycan matrix and calcification of bone matrix. When MSCs undergo osteogenic differentiation, they express osteoblast markers. MSCs undergo osteogenic differentiation in a progressive manner. ${ }^{35}$ The early phase of in vitro osteogenic differentiation can be characterized by the expression of alkaline phosphatase (ALP) at mRNA and protein level. ${ }^{36}$ After the initial peak of ALP expression, its level starts to decrease. Late stage of osteogenic differentiation results in elevated expression of osteopontin, followed by calcium and phosphate deposition. ${ }^{35,37}$ Thus, early phase of osteogenic differentiation was analyzed with ALP assay, and the amount of calcium deposition was assayed with alizarin red staining for detection of late phase differentiation. Compared with epitope free scaffold and bare surface, ALP activity of rMSCs on TN-C mimetic scaffold was increased significantly by about 2 -fold on day 3. Although, ALP activity of rMSCs on biologically active scaffold displayed lower ALP activity on day 7 compared with that on day 3, ALP 
(a)

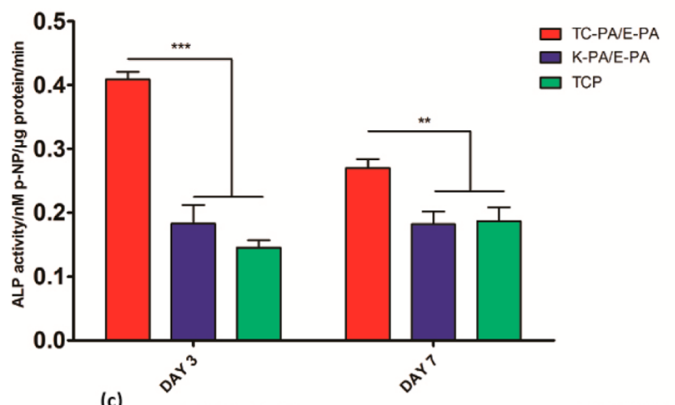

(b)

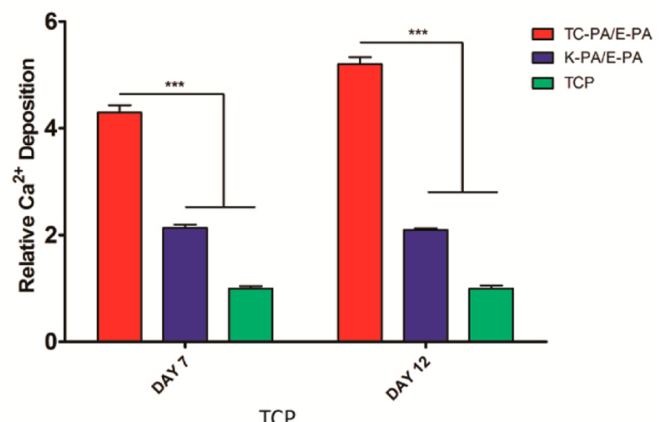

(c) TC-PA/E-PA
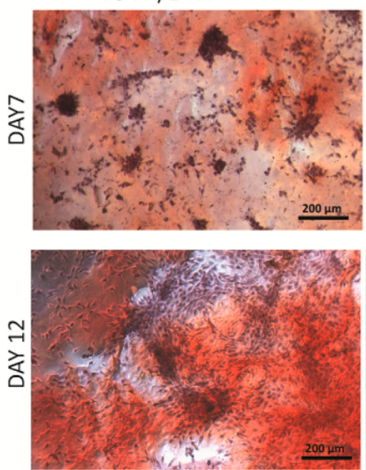

K-PA/E-PA
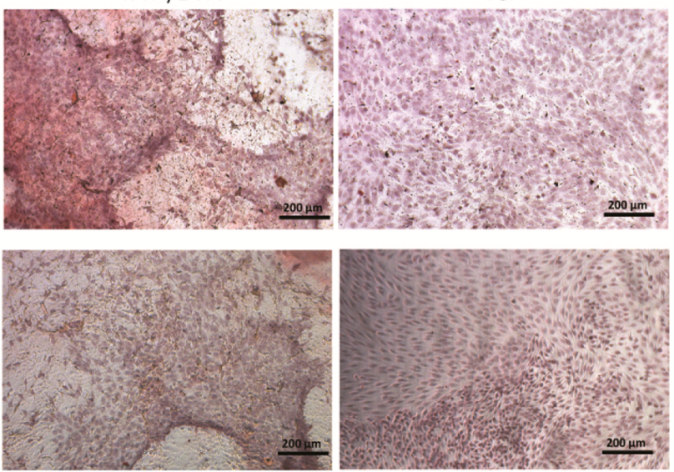

Figure 6. Effect of PA nanofibers on osteogenic activity. (a) ALP activity of rMSCs on day 3 and 7. (b) Quantification of relative calcium deposition on day 7 and 12. (c) Deposition of calcium on peptide coated substrates and TCP on day 7 and 12 as demonstrated by alizarin red staining; $* * *<<$ $0.001, * * p<0.01$.

activity of cells on bioactive surface still remained higher than those on epitope free scaffold and TCP (Figure 6a). In order to test the mineral deposition of rMSCs as a late marker of osteogenic differention, alizarin red staining was performed on day 7 and day 12, and calcium deposition was quantified by extracting alizarin red bound calcium from the surface followed by a colorimetric measurement. The results indicated that calcium deposition on TC-PA/E-PA was significantly higher compared with that on K-PA/E-PA surface and bare surface on day 12. Similar mineral deposition pattern was also observed on day 7 (Figure 6b,c).

The differentiation of MSCs in vitro largely depends on the culture conditions. Osteogenic differentiation of MSCs is generally induced by the presence of dexamethasone, ascorbic acid, and $\beta$-glycerol phosphate. ${ }^{4}$ However, TN-C mimetic peptide scaffold can induce osteogenic differentiation despite the absence of these constituents or supplemental osteogenic growth factors. TN-C derived bioactive epitope acted as the sole triggering factor for osteogenic commitment of MSCs, since ALP activity, calcium deposition, and expression of osteogenic marker genes were higher on TN-C mimetic peptide nanofibers compared with epitope free control peptide nanofibers and bare surface. Although the presence of a stiff environment is also known to be an important requirement for induction of osteogenic differentiation, ${ }^{9-11}$ bioactivity caused by $\mathrm{TN}-\mathrm{C}$ derived peptide sequence is more effective than the requirement of high modulus to induce osteogenic differentiation. Therefore, bioactivity introduced by our PA scaffold is the only factor to induce osteogenic differentiation compensating for the lack of osteogenic supplements in the medium and rigidity of the substrate.

\section{CONCLUSION}

Bone tissue regeneration is essential for the treatment of large bone defects. Alternative to the current treatments, which have several drawbacks including infection, chronic pain, and stiffness, using biomaterial scaffolds that can mimic the microenvironment of osteogenic cells has become highly promising for clinical therapy in recent years since these biomaterials can provide key bioactive signals to create a synthetic microenvironment to control cell fate. In this study, we presented that a single bioactive epitope, TN-C mimetic peptide, enables MSCs to (1) attach and adapt to the bioactive gel, (2) go through a morphological change that is supported with a change in the gene expression profile as a sign of differentiation, (3) display increased ALP activity, and (4) deposit calcium and form a mineralized matrix. The results show that the extracellular mimetic approach used here allows MSCs to undergo the osteogenic differentiation pathway without any additional factors including growth factor and medium supplements and regardless of suitable mechanical properties normally required for osteogenic differentiation. Overall, our results showed that incorporation of tenascin-C derived bioactive epitope into a self-assembled peptide amphiphile nanofiber system constitutes a promising platform for bone formation by providing a suitable microenvironment for osteogenic differentiation.

\section{ASSOCIATED CONTENT}

\section{S Supporting Information}

LC-MS measurements, phase contrast image of rMSCs, gene expression levels of different differentiation markers, and primer list used for qRT-PCR expression analysis. This material is available free of charge via the Internet at http://pubs.acs.org. 


\section{AUTHOR INFORMATION}

\section{Corresponding Authors}

* E-mail: moguler@unam.bilkent.edu.tr (M.O.G).

*E-mail: atekinay@unam.bilkent.edu.tr (A.B.T.).

Notes

The authors declare no competing financial interest.

\section{ACKNOWLEDGMENTS}

We thank Z. Erdogan, M. Guler, and Z. E. Ulger for their technical help. This work was funded by Scientific and Technological Research Council of Turkey (TUBITAK) Grant No. 111M410. M.S. is supported by TUBITAK-BIDEB (2211) Ph.D. fellowship. M.O.G. and A.B.T. acknowledge support from the Turkish Academy of Sciences Distinguished Young Scientist Award (TUBA-GEBIP).

\section{ABBREVIATIONS}

MSC, mesenchymal stem cell; ECM, extracellular matrix; TN, tenascin; FNIII, fibronectin type III; PA, peptide amphiphile; DMF, dimethylformamide; DCM, dichloromethane; LC-MS, liquid chromatography-mass spectrometry; SEM, scanning electron microscope; STEM, scanning transmission electron microscope; CD, circular dichroism; qRT-PCR, quantitative real-time polymerase chain reaction; ALP, alkaline phosphatase

\section{REFERENCES}

(1) Bruder, S. P.; Fink, D. J.; Caplan, A. I. J. Cell. Biochem. 1994, 56, 283-294.

(2) Caplan, A. I. J. Orthop. Res. 1991, 9, 641-650.

(3) Li, W. J.; Tuli, R.; Huang, X.; Laquerriere, P.; Tuan, R. S. Biomaterials 2005, 26, 5158-5166.

(4) Jaiswal, N.; Haynesworth, S. E.; Caplan, A. I.; Bruder, S. P. J. Cell. Biochem. 1997, 64, 295-312.

(5) Boyan, B. D.; Hummert, T. W.; Dean, D. D.; Schwartz, Z. Biomaterials 1996, 17, 137-146.

(6) Bullett, N. A.; Bullett, D. P.; Truica-Marasescu, F. E.; Lerouge, S.; Mwale, F.; Wertheimer, M. R. Appl. Surf. Sci. 2004, 235, 395-405.

(7) Long, S. F.; Clarke, S.; Davies, M. C.; Lewis, A. L.; Hanlon, G. W.; Lloyd, A. W. Biomaterials 2003, 24, 4115-4121.

(8) Nelea, V.; Luo, L.; Demers, C. N.; Antoniou, J.; Petit, A.; Lerouge, S.; M, R. W.; Mwale, F. J. Biomed. Mater. Res., Part A 2005, 75, 216-223.

(9) Engler, A. J.; Sen, S.; Sweeney, H. L.; Discher, D. E. Cell 2006, $126,677-689$.

(10) Huebsch, N.; Arany, P. R.; Mao, A. S.; Shvartsman, D.; Ali, O. A.; Bencherif, S. A.; Rivera-Feliciano, J.; Mooney, D. J. Nat. Mater. 2010, 9, 518-526.

(11) Shih, Y. R.; Tseng, K. F.; Lai, H. Y.; Lin, C. H.; Lee, O. K. J. Bone Miner. Res. 2011, 26, 730-738.

(12) Gehron Robey, P. Endocrinol. Metab. Clin. North Am. 1989, 18, 858-902.

(13) Mackie, E. J.; Tucker, R. P. J. Cell Sci. 1992, 103 (Pt 3), 765771.

(14) Jones, F. S.; Jones, P. L. Dev. Dyn. 2000, 218, 235-259.

(15) Pearson, C. A.; Pearson, D.; Shibahara, S.; Hofsteenge, J.; Chiquet-Ehrismann, R. EMBO J. 1988, 7, 2977-2982.

(16) Alford, A. I.; Hankenson, K. D. Bone 2006, 38, 749-757.

(17) Mackie, E. J.; Thesleff, I.; Chiquetehrismann, R. J. Cell Biol. 1987, 105, 2569-2579.

(18) Vakeva, L.; Mackie, E.; Kantomaa, T.; Thesleff, I. Anat. Rec. 1990, 228, 69-76.

(19) Mackie, E. J.; Ramsey, S. J. Cell Sci. 1996, 109 (Pt 6), 15971604.

(20) Meiners, S.; Nur-E-Kamal, M. S. A.; Mercado, M. L. T. J. Neurosci. 2001, 21, 7215-7225.

(21) Cui, H.; Webber, M. J.; Stupp, S. I. Biopolymers 2010, 94, 1-18.
(22) Dvir, T.; Timko, B. P.; Kohane, D. S.; Langer, R. Nat. Nanotechnol. 2011, 6, 13-22.

(23) Hartgerink, J. D.; Beniash, E.; Stupp, S. I. Science 2001, 294, $1684-1688$.

(24) Sargeant, T. D.; Guler, M. O.; Oppenheimer, S. M.; Mata, A.; Satcher, R. L.; Dunand, D. C.; Stupp, S. I. Biomaterials 2008, 29, 161171.

(25) Sargeant, T. D.; Oppenheimer, S. M.; Dunand, D. C.; Stupp, S. I. J. Tissue Eng. Regen. Med. 2008, 2, 455-462.

(26) Silva, G. A.; Czeisler, C.; Niece, K. L.; Beniash, E.; Harrington, D. A.; Kessler, J. A.; Stupp, S. I. Science 2004, 303, 1352-1355.

(27) Ceylan, H.; Kocabey, S.; Tekinay, A. B.; Guler, M. O. Soft Matter 2012, 8, 3929-3937.

(28) Kocabey, S.; Ceylan, H.; Tekinay, A. B.; Guler, M. O. Acta Biomater. 2013, 9, 9075-9085.

(29) Stanford, C. M.; Jacobson, P. A.; Eanes, E. D.; Lembke, L. A.; Midura, R. J. J. Biol. Chem. 1995, 270, 9420-9428.

(30) Niece, K. L.; Hartgerink, J. D.; Donners, J. J.; Stupp, S. I. J. Am. Chem. Soc. 2003, 125, 7146-7147.

(31) Pevsner-Fischer, M.; Levin, S.; Zipori, D. Stem Cell Rev. 2011, 7, $560-568$.

(32) Chang, H. H.; Hemberg, M.; Barahona, M.; Ingber, D. E.; Huang, S. Nature 2008, 453, 544-547.

(33) Wright, E.; Hargrave, M. R.; Christiansen, J.; Cooper, L.; Kun, J.; Evans, T.; Gangadharan, U.; Greenfield, A.; Koopman, P. Nat. Genet. 1995, 9, 15-20.

(34) Yamashiro, T.; Wang, X. P.; Li, Z.; Oya, S.; Aberg, T.; Fukunaga, T.; Kamioka, H.; Speck, N. A.; Takano-Yamamoto, T.; Thesleff, I. J. Bone Miner. Res. 2004, 19, 1671-1677.

(35) Huang, Z.; Nelson, E. R.; Smith, R. L.; Goodman, S. B. Tissue Eng. 2007, 13, 2311-2320.

(36) Aubin, J. E. Rev. Endocr. Metab. Disord. 2001, 2, 81-94.

(37) Hoemann, C. D.; El-Gabalawy, H.; McKee, M. D. Pathol. Biol. (Paris) 2009, 57, 318-323.

(38) Smith, C. K.; Withka, J. M.; Regan, L. Biochemistry 1994, 33, 5510-5517.

(39) Toksoz, S.; Mammadov, R.; Tekinay, A. B.; Guler, M. O. J. Colloid Interface Sci. 2011, 356, 131-137.

(40) Aumailley, M.; Gayraud, B. J. Mol. Med. (Berlin) 1998, 76, 253265 . 